\title{
FOCUS AWARDS 2002
}

(DH) Depertanent
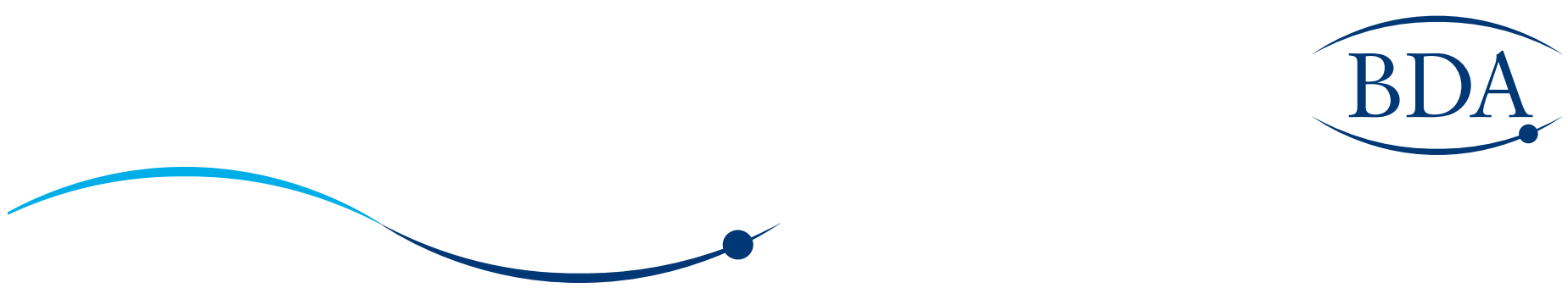

The Lindley Dental Practice was a finalist at the joint BDA/DoH Focus Awards. What does this practice do that made it stand out from the other applicants?

The services provided by the Lindley Dental Centre were described by the Chairman of the BDA's Executive Board, John Renshaw as providing "patient interactivity with a degree of patient control'; it is for their careful implementation of some simple ideas that took them to the final of the Focus Awards 2002.

The Lindley practice gives its patients this 'interactive control' in several ways:

- Carrying out patient surveys and acting on the results

- Having an informative, interactive website

- Providing a series of patient leaflets reflecting patient needs

- Educating patients, empowering them to help themselves

\section{PATIENT SURVEY AND ACTING ON THE RESULTS}

The staff at the Lindley practice wanted to know what their patients thought of their experiences at the practice, so to find out, they held a survey. The patients were asked about their visits to the practice and the

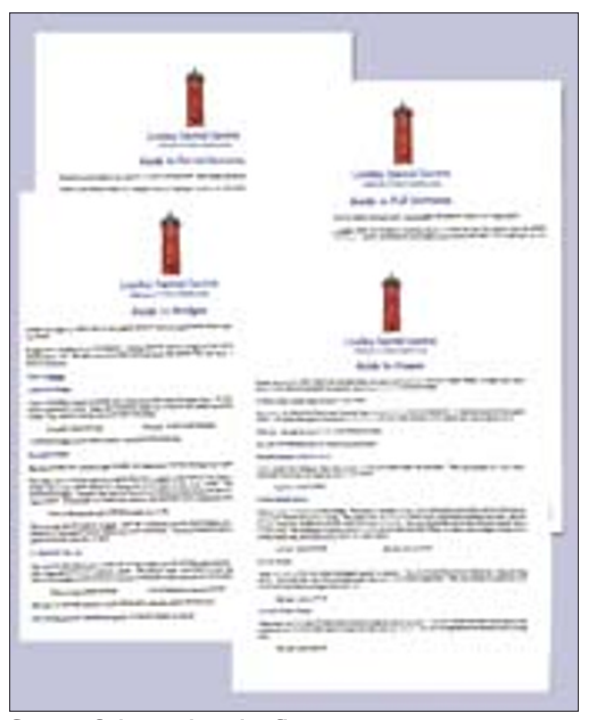

Some of the patient leaflets.

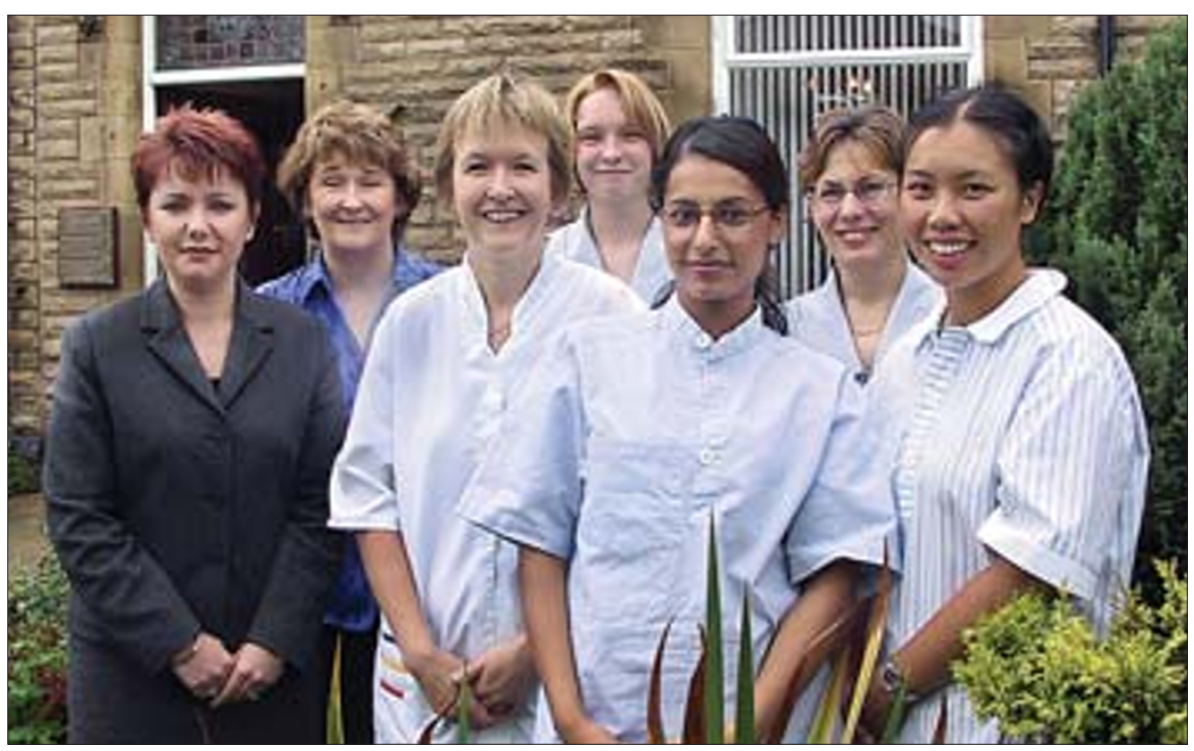

The team at Lindley Dental Practice.

results showed that the patients were all very happy with the service and treatments. However, there is always room for improvement and two points came up as areas for some work.

The first was that the practice's patient leaflet series was so well valued that the patients wanted more, specifically on cosmetic dentistry and one on endodontics. Sharon Jardine, practice principal, wrote these new leaflets and they are now available at the practice and on the website.

The second point that resulted from the survey was about treatment costs. Patients wanted to be better informed of the potential costs of their care. The practice installed a computerized system that can produce a printed estimate for the cost of treatments. Having this gives the patients a feeling of control and involvement in the decisionmaking regarding their own treatment.

Another patient survey is planned soon. This will let Sharon and her team know if they are still getting things right or if their patients still want more.

\section{INFORMATIVE INTERACTIVE WEBSITE}

The practice wesbite has many useful functions; two particularly effective ones are the emergency information page and the online appointments booking facility:

\section{Emergency information page}

This part of the website helps patients to work out if their dental discomfort is an actual emergency. A series of common oral ailments are listed with advice on what the patient should do if they are suffering from it. It also tells them what they can do if they still think that they have an emergency situation.

\section{Online appointments}

Patients can send a request from the website for their next appointment time. It is not always possible to give the patient the time that they requested but it gives them the chance to request ones that are convenient for them. Even people who are not registered with the practice can request their first appointment through the web- 


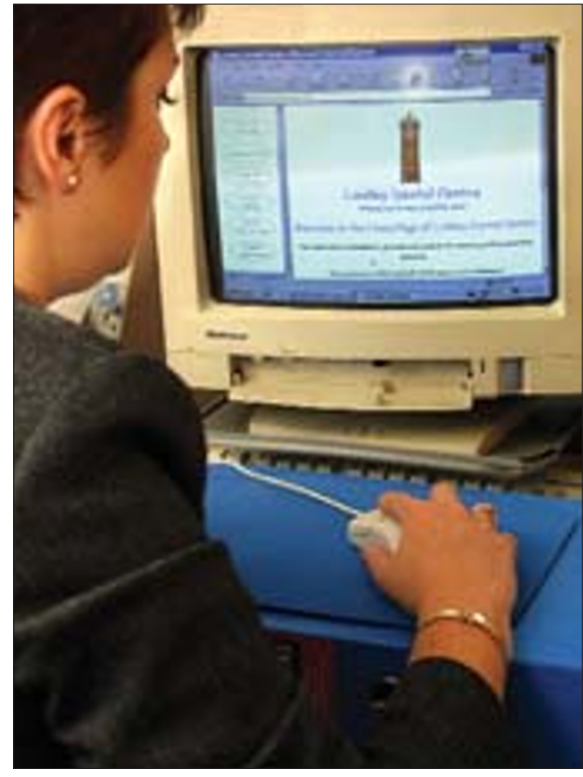

Using the website.

site, while regular attendees can request an appointment with their own dentist.

The website is proving a popular addition to the practice's services and it is regularly kept up to date, so people know it is worth going back to.

The Lindley practice website is a clear and simple, yet effective way of making

\section{How the practices got involved}

In order to participate, each entrant had to submit a nomination form specifying the innovations under the categories given below. Any practice that had applied last year was not allowed to use the same innovations in their application.

information on the practice as accessible as possible. The surgery contact details are on the site, along with the series of patient information leaflets described below. This way patients have access to reliable information about treatment options from home.

\section{PATIENT LEAFLETS SERIES REFLECTING PATIENT NEEDS}

The practice has produced a series of leaflets for their patients to read which covers most of the techniques used at the surgery. The patient leaflets were written by Sharon Jardine, with an associate (who has since left the practice). With these the patients can take something away to read about the treatments that have been recommended.

Guides on cosmetic dentistry and endodontics are the titles most recently added to the list, and were written in response to the results of the patient survey mentioned above. These recent additions illustrate that patients have an input to many aspects of their dental practice.

\section{EMPOWERING PATIENTS}

It seems the real secret to this practice's success in the Focus Awards is the attitude of the whole dental team towards
- Patients' experiences contacting the practice/clinic before, during and after treatment.

- Patients' experiences whilst in the reception or waiting area

- Patients' experiences during clinical treatment

- General communication with the patients

- Any other patient-focused innovation

The Focus Awards is a joint initiative between the Department of Health of England and the British Dental Association. It was set up to recognise and celebrate the innovative patient-focused projects being carried out across England.

the importance of really involving patients in decisions. This attitude means that the practice not only looks at ways of trying to provide a genuine patient-focus, by making the systems within the practice work for the patients and the practice, but also takes action on patients" suggestions. This is best illustrated in the quick provision of extra patient leaflets. In fact the element of control is just as important in patient focus, as seen here with the internetbased appointment system.

The practice also ensures that patients continue to receive information, especially through the web site which means the information is available all the time and patients can access it without having to visit or telephone. As the Lindley Practice has demonstrated so effectively, 'patient interactivity with a degree of patient control' really does work.

Naomi Davis

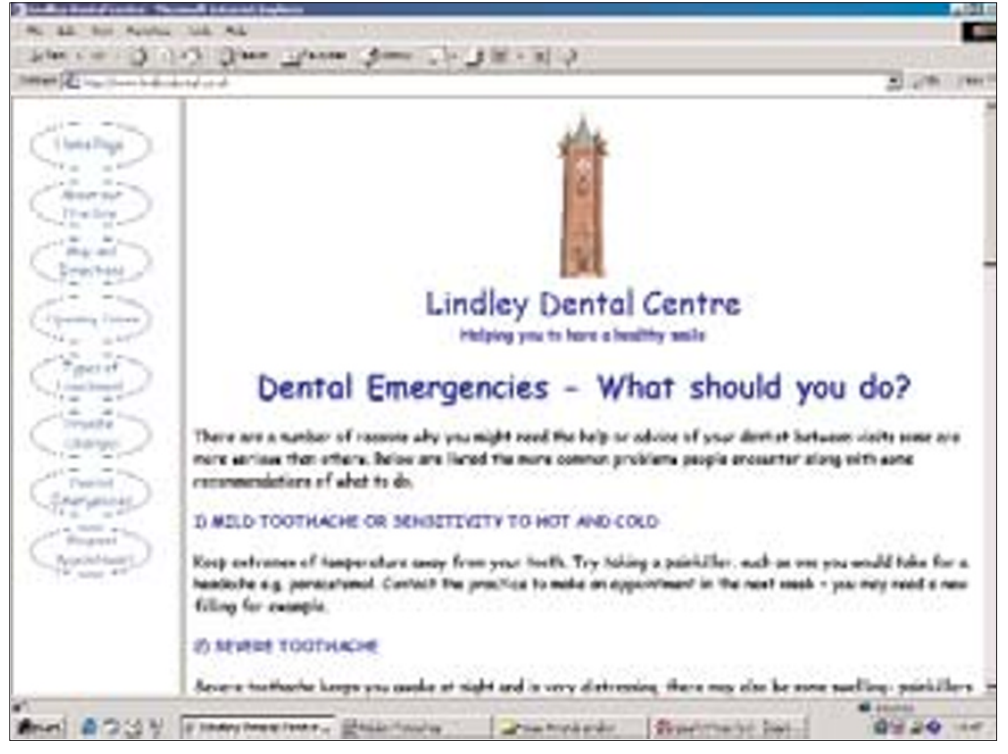

The emergency page from the Lindley practice website.

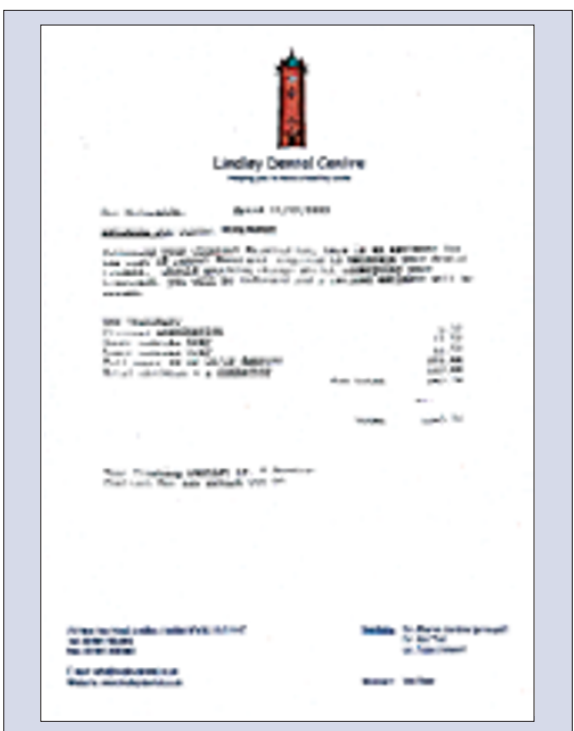

An example of an estimate. 